\title{
Traducción de la variación lingüística: una visión diacrónica
}

\section{(Translation of linguistic variation: a diachronic approach)}

\author{
ISABEL TELLO FONS \\ Eiee2002us@yahoo.com \\ Universitat Jaume I
}

Fecha de recepción: 27 de julio de 2012

Fecha de aceptación: 15 de octubre de 2012

Resumen: A medida que pasa el tiempo, las traducciones van adaptándose a nuevos acontecimientos históricos, tendencias traductológicas y corrientes literarias. En este trabajo se presenta un breve estudio sobre la relación entre el paso del tiempo y la traducción de uno de los problemas clásicos de la Traductología: la traducción de la variación lingüística. A través de dos conocidas novelas de la literatura inglesa del siglo XIX, se realiza un acercamiento diacrónico a las técnicas de traducción del dialecto en literatura y a las circunstancias que puedan haber influido en estas decisiones traductoras.

Palabras clave: Traducción literaria. Técnica de traducción. Norma traductora. Dialecto. Diacronía.

Abstract: Over the course of time, translations adapt to new historical sceneries, translation theories, and literary trends. This short study aims to analyze how the course of time influences one of the classical problems in Translation Studies: the translation of linguistic variation. Using two well-known English novels from the XIX century, this paper not only focuses on the translation techniques put into practice diachronically but on the factors which may have conditioned the translation decisions.

Key words: Literary Translation. Translation Technique. Translation Norm. Dialect. Diachrony.

\section{INTRODUCCIÓN}

Según Hurtado ${ }^{1}$ La variación lingüística hace referencia a las variedades funcionales de la lengua que tienen que ver tanto con la persona que la utiliza como con el contexto de uso particular de la misma. Hoy en día, no se puede negar la importancia de la variación lingüística que

\footnotetext{
${ }^{1}$ HuRTAdo AlBIR, Amparo. Traducción y traductología. Introducción a la traductología. Madrid: Cátedra, 2001, p. 544.
} 
muestra un buen número de obras de ficción narrativa y que se traduce en la diversidad de voces como elemento que confiere identidad a la obra en cuestión.

La teoría de los polisistemas de Even-Zohar ${ }^{2}$ coloca la traducción de literatura dentro de un polisistema o conjunto de componentes de una sociedad. En el presente artículo se van a estudiar las tensiones intrasistémicas (según la terminología de esta teoría) que hacen referencia a las relaciones dentro del polisistema literario español, en este caso centradas en el tiempo y en el transcurso de éste con relación a las traducciones. Dicho de otra manera, se intentará dar cuenta de cómo el paso del tiempo afecta a la forma de traducir la variación lingüística en literatura. Así, se abordará un breve análisis sobre la traducción de este fenómeno y, en concreto, de la traducción del dialecto, a lo largo de un periodo de tiempo a través de las obras inglesas Wuthering Heights y Hard Times, escritas por Emily Brontë y Charles Dickens respectivamente. La comparación de estas dos obras, aunque insuficiente, puede ofrecer pistas interesantes sobre las normas de uso relativas a la traducción del dialecto en momentos históricos distintos y explicar los cambios que se puedan haber producido, o no, en cuanto a opciones traductoras. Los hallazgos en este sentido podrían ser útiles tanto para comprender la evolución de las técnicas de traducción del dialecto a lo largo de un período de tiempo y hasta nuestros días como para entender mejor la historia del polisistema literario español y, más concretamente, de la literatura traducida.

Wuthering Heights y Hard Times son dos novelas inglesas que hoy en día forman parte de la literatura universal. Estas dos obras poseen una enorme repercusión como producto literario y, a consecuencia de ello, son numerosas las traducciones que desde su publicación se han vertido al castellano. Los fragmentos que se incluyen en este trabajo son parte de las traducciones realizadas en los siglos XX y XXI y los personajes que se estudian hacen uso del dialecto para expresarse. La influencia de los hechos históricos, sociales, políticos y culturales españoles de la época sobre la forma de traducir, así como el influjo de la procedencia de las obras, son temas en los que es interesante indagar para contextualizar y entender tanto el análisis de este trabajo como sus resultados. La atención se centrará, sobre todo, en averiguar a través de las técnicas de traducción utilizadas qué norma traductora ${ }^{3}$ rige el comportamiento de los traductores. Tras la observación de las técnicas de traducción, se abre el debate sobre si éstas siguen la estela de otras traducciones ya realizadas y, por lo tanto, se inscriben en tendencias de traducción muy definidas, o si, por el contrario,

2 EVEN-ZOHAR, Itamar. "Polysystem Studies", en Poetics Today, 11: 1, 1990

${ }^{3}$ TOURY, Gideon. Los estudios descriptivos de traducción y más allá. Metodología de la investigación en estudios de traducción. Madrid: Cátedra, 2004. 
son elecciones individuales de los traductores propiciadas por distintos motivos.

\section{LAS TÉCNICAS DE TRADUCCIÓN DEL DIALECTO}

Las técnicas de traducción del dialecto propuestas por los diferentes autores son numerosas. Elegir una $u$ otra depende de los condicionamientos externos que atañen a cualquier decisión traductora como son los requisitos impuestos por el encargo de traducción, las características de los lectores que vayan a recibir el texto y la finalidad de la traducción y el estatus que ésta tendrá en la cultura de llegada.

En 1965, Catford ${ }^{4}$ da unos consejos para traducir las distintas variedades de la lengua que clasifica en su trabajo. De este modo:

1) No siempre será necesaria la traducción del idiolecto, puesto que la forma de hablar del emisor del texto no siempre es un rasgo importante en la situación. Cuando este emisor sí sea relevante y el idiolecto identifique su carácter, sí podrá traducirse por medio de rasgos idiolectales "equivalentes".

2) Los dialectos estándar, considerados como no marcados, se traducirán a la lengua meta por otro dialecto estándar. El autor apunta que cuando la lengua meta no tenga un dialecto estándar habrá de crearse uno o seleccionar un dialecto particular (más tarde, el autor aclara que este problema surge en la traducción de la Biblia a lenguas todavía no constituidas formalmente).

3) Para los dialectos geográficos, el traductor primará la geografía humana antes que la topográfica. La equivalencia en estos casos debe ser funcional, y por lo tanto, el dialecto Cockney (dialecto del sureste de Inglaterra) se traducirá a la lengua francesa por el Parigot (dialecto del norte de Francia) por tratarse ambos de dialectos urbanos. Sobre este ejemplo, Catford avisa de las probables inequivalencias entre dialectos cuyos marcadores son básicamente fonológicos (Cockney) o léxicos (Parigot).

4) En cuanto a los états de langue o dialectos temporales arcaicos, la equivalencia total en el tiempo en la traducción a la LM no es, según el autor, posible ni deseable. Sí acepta un "sabor arcaico" a través, por ejemplo, de marcadores léxicos o fonológicos.

Slobodník ${ }^{5}$ parte de la idea de que la traducción es una forma específica de comunicación y destaca la relación con el destinatario o lector

${ }^{4}$ CATFORD, John C., A Linguistic Theory of Translantion. London: OUP, 1965, pp. 86-92.

5 SLOBODNík, Dusan, "Remarques sur la traduction des dialects", en Holmes, J. (ed.), The Nature of Translation, Essays on the Theory and Practice of Literary Translation, 1968, Bratislava, Publishing House of the Slovak Academy of Sciences, pp. 139-143. 
que determina y modifica la estructura de la traducción. El autor cree que se puede alcanzar la noción de "homología de la funcionalidad" buscando una solución teórica para la traducción de los elementos dialectales. Los rasgos dialectales pueden presentarse en las obras literarias en tres formas:

1) en el discurso indirecto (es decir, en el discurso del autor), que sería el uso de palabras dialectales en los textos originales para designar hechos u objetos de la vida. Considerando que en este caso son mayormente los sustantivos los que representan esos elementos dialectales, Slobodník propone la traducción por medio de elementos también dialectales en la LM. No recomienda que se haga lo mismo cuando se trate de verbos que sirven para describir una acción y sí admite que un elemento dialectal pueda traducirse por otro no dialectal porque el efecto final no se consigue a través de palabras aisladas sino a través de toda la información semántica y estética del texto completo;

2) en el discurso directo de algunos personajes. Esos rasgos dialectales caracterizan al personaje en el plano espacial y social. Lo que propone el autor es acentuar el estilo de la lengua hablada (con elementos interdialectales), así como recurrir a la sustitución. De esta forma, la "homología de la funcionalidad" de la que habla se verá poco alterada, ya que la lengua hablada estaría a medio camino entre la lengua escrita y el dialecto;

3) en el discurso directo de algunos personajes, donde el autor busca caracterizarlos desde el punto de vista social y en la mayoría de los casos pretende crear un efecto cómico. En este caso, se podrían utilizar elementos análogos de un dialecto de la LM. Lo más importante es reproducir el efecto deseado por el autor. Aquí el color local del original no tendría importancia según el autor.

Para la traducción del dialecto geográfico, House $^{6}$ pone el ejemplo del dialecto Hiberno-English (el inglés que utilizan los irlandeses), cuya solución sería buscar un dialecto equivalente en cuanto al tamaño de ciudad y clase social. El dialecto temporal tendría que sustituirse por un texto arcaizante. traducción:

Gregory, en un artículo de $1980^{7}$, da algunas propuestas de

- piensa que los idiolectos no tienen por qué traducirse siempre, puesto que la identidad del emisor puede no ser relevante en el

\footnotetext{
${ }^{6}$ HousE, Juliane. A Model for Translation Quality Assessment. Tübingen: TBL Verlag Gunter Narr, 1977.

${ }^{7}$ GREGORY, Michael, artículo de 1980 en Hatim, Basil e lan Mason. Translation. An Advanced Resource Book. London: Routledge, 2004, pp. 188-189.
} 
ámbito situacional. No lo sería, por ejemplo, en textos científicos u oficiales, pero sí en novelas y obras de teatro. Cita el ejemplo de Shakespeare o Dickens, cuyos personajes en muchas ocasiones están marcados por un idiolecto que los autores crearon para ellos. El traductor tiene la responsabilidad de intentar distinguir la forma de hablar de estos personajes en la LM, aunque las marcas de esta individualidad no serán similares en ambas lenguas;

- en cuanto a la traducción de un dialecto que aparece en pasajes de un texto estándar, propone que el traductor seleccione un dialecto equivalente en la LM, pero recomienda el uso del sentido común.

Para sus categorías de idiolecto, registro y jerga, Newmark ${ }^{8}$ propone en su obra de 1988 varios procedimientos de traducción, introduciendo la categoría de dialecto y, dentro de éste, el argot:

- no le parece razonable traducir el dialecto francés de un minero de una novela de Zola por el dialecto de un minero galés al traducirlo al inglés. Para ello, el traductor debería estar muy familiarizado con el dialecto de Gales. El autor expone el riesgo al que se enfrentan los traductores a parecer anticuados debido a la poca popularidad de los dialectos en la lengua inglesa actualmente;

- propone traducir el idiolecto en documentos escritos por autores importantes, pero no dice cómo;

- si el registro se aparta de forma extrema del lenguaje educado estándar, el traductor "puede apartarse de su empresa de mantener la equivalencia funcional y producir una traducción de información, un tipo de estilo indirecto";

- puesto que existe una gran variedad de edades, periodos y clases sociales en el argot, los problemas de su traducción difícilmente se prestan a la generalización. El traductor puede escoger entre la transcripción, que aporta a su versión el color local, y la traducción literal, la cual, si hay solapamiento cultural, hace comprensible la metáfora. En casos aislados el argot se traduce de forma similar a la de la metáfora, teniendo presente que, salvo para eventos corrientes, los equivalentes son raros para el argot y el traductor puede tener que "meterlo donde pueda".

En su trabajo de 1991 sobre la equivalencia en traducción, Rabadán ${ }^{9}$ da algunos consejos a propósito de la clasificación dialectal que hace:

\footnotetext{
${ }^{8}$ NeWmark, Peter, A Textbook of Translation, London: Prentice Hall, 1988, p. 195.

${ }^{9}$ RABADÁN, Rosa, Equivalencia y traducción: problemática de la equivalencia translémica inglés-español. León: Universidad, 1991, pp. 83-96.
} 
1) Para los textos escritos completamente en dialecto considera que en estos casos nunca debe traducirse hacia otro dialecto sino hacia la lengua estándar, pudiendo añadir en la traducción "dijo en dialecto".

2) En cuanto a las variantes diacrónicas: aquí la autora aboga por no traducir los rasgos temporales, pues cree que en el texto marcado temporalmente ya existen "otros indicadores (al margen de la estructura superficial) que lo caracterizan como perteneciente al estadio diacrónico".

Al mismo tiempo, al hablar de la variante dialectal geográfica, la autora pone de manifiesto su disconformidad con la restitución al castellano de este tipo de variante por otro dialecto de la lengua española, y lo ejemplifica con la novela de D. H. Lawrence Lady Chatterley's Lover, donde uno de los personajes utiliza el habla de la zona inglesa de Yorkshire. La postura de Rabadán sobre la traducción dialectal es escéptica, pues considera que ésta no será aceptada por los lectores meta.

Hatim y Mason ${ }^{10}$ hacen hincapié en la importancia de esta cuestión en cada tipo de dialecto poniendo algún ejemplo:

a) destacan los problemas de comprensión que ocasionan, incluso para los lectores de los textos originales, los dialectos temporales y la dificultad para los traductores de estar al día de todos los cambios que acontecen en la lengua. Proponen dos opciones: la traducción hacia una variante arcaica de la LM o hacia la variante contemporánea;

b) tanto para el dialecto geográfico como para el social sostienen la importancia de los aspectos sociales, ideológicos y políticos que se puedan derivar de ellos. De esta forma, destacan la función sociocultural que algunos dialectos geográficos pueden tener (mayor o menor consideración de ciertos dialectos) e intentan concienciar a los traductores de la importancia de las implicaciones políticas e ideológicas que puedan conllevar. Aun así, sostienen que el principio de equivalencia exigiría una traducción que recogiera toda la carga social del original, aunque no dicen cómo hacerlo. También cuestionan que el idiolecto se pueda traducir, ya que le otorgan un significado social.

En su trabajo de $1997^{11}$, sí profundizan algo más en los problemas que puede acarrear la traducción de los idiolectos en los textos literarios y

\footnotetext{
${ }^{10}$ HATIM, Basil e lan MASON. Teoría de la traducción. Una aproximación al discurso. Barcelona: Ariel, 1995, p. 60.

${ }^{11}$ HATIM, Basil e lan MASON. Translator as Communicator, London: Routledge, 1997.
} 
ofrecen la equivalencia pragmática como solución, que pasaría por incluir elementos subestándar en la LM.

Muñoz ${ }^{12}$ hace una distinción importante refiriéndose a los dialectos sociales y geográficos: si los rasgos dialectales son intencionados por parte de los emisores, se considera que ejercen una función concreta en el texto y que reflejan el estilo de los mismos. En este caso, "es necesario asignarles un significado, para lo que se recurre a la gama de estereotipos de la comunidad lingüística en cuyo seno se crean. Qué hacer con esa información es algo que pertenece a la estrategia particular de cada profesional". Si por el contrario los rasgos dialectales se consideran como no intencionales (reflejan el idiolecto de los emisores), estos rasgos no se suelen reflejar en la traducción porque las variedades geográficas y sociales solo tienen sentido en la lengua en que se dan y los textos traducidos reflejan los idiolectos de los mediadores. Cuando se trata de dialectos temporales, Muñoz afirma que aun en el caso de querer traducir intentando reproducir la intención del autor, la lectura estará mediatizada por la perspectiva contemporánea del traductor y del público a quien se destina.

Julià $^{13}$ está a favor de la traducción de las variantes dialectales por otras variantes dialectales de la LM. Este autor rebate los argumentos en contra de la traducción de las variantes geográficas y sociales, que encuentra poco sólidos puesto que, aunque son coherentes desde el punto de vista social y geográfico no lo son desde el lingüístico. Aun así, no rechaza otras posibilidades según los casos y reconoce que la traducción dialectal será más fácil en algunas ocasiones que en otras. Afirma que esta opción podrá realizarse dependiendo de algunas consideraciones:

1) que la presencia dialectal pueda aparecer en diferentes géneros textuales (de narrativa, teatro, etc.) y con diversos usos textuales. Habrá que contemplar, pues, la especificidad de cada caso concreto; 2) que hay que tener en cuenta la función social del dialecto en el texto original (con qué finalidad lo usa el autor) y que esta función puede ser de muy diverso tipo (añadir color local, diferenciar socialmente, marcar procedencia geográfica, etc.);

3) que cada lengua tiene una especificidad dialectal. Existen lenguas con más dialectos y otras con menos, lenguas más reacias al uso dialectal y otras más tolerantes, lo cual hará más o menos viables las traducciones dialectales. A ello hay que añadir las connotaciones sociológicas que suelen adscribirse a los dialectos (con los tópicos que pueden conllevar);

\footnotetext{
${ }^{12}$ MUÑOz MARTín, Ricardo, Lingüística para traducir, Barcelona, Teide, 1995, pp. 5-42.

${ }^{13}$ JULIÀ BALLBĖ, Josep. "Dialectes i traducció: reticències i aberracions", en Bacardí, M. (ed.), Actes del II Congrès Internacional sobre Traducció, Abril 1994, Bellaterra, Servei de Publicacions de la Universitat Autónoma de Barcelona, 1997, pp. 561-574.
} 
4) que hay que poder combinar la presencia dialectal con la verosimilitud de una traducción, sin generar extrañezas o artificialidades derivadas de las connotaciones sociológicas adscritas a cada dialecto. De todos modos, según Julià, son problemas que la ficción y la tradición lectora pueden contribuir a superar y cuya gravedad depende de cada ámbito lingüístico. La aceptación de marcas dialectales (geográficas) en una traducción depende de la tradición traductora instaurada en una cultura y de la aceptación de los lectores;

5) que no hay una única opción válida para resolver la presencia dialectal geográfica, sino que al traductor se le abren diferentes soluciones (geográficas, sociales, interdialectales) que tiene que sopesar.

Dentro del contexto catalán, propone utilizar recursos catalanes reales, sobre todo cuando se trate de casos en los que no haya adaptación cultural global. Así se conseguirá un alto grado de naturalidad y al mismo tiempo, se ampliarán las expectativas de los lectores.

Mayoral ${ }^{14}$ es consciente de que una propuesta de modelo de proceso de traducción de la variación tendría que comulgar con demasiados preceptos de las diferentes corrientes de traducción para ser útil, por lo que acude a las máximas de Grice (1975: 41-58) que le permitirían explicar "una variedad de situaciones comunicativas de traducción distintas y de soluciones dispares (...)". De esta forma, se sirve de esas máximas para determinar qué criterios tendría que seguir un traductor a la hora de verter la variante dialectal a otra lengua:

- Ajustarse al contexto y la situación promovidos por el encargo de traducción (máxima de calidad y máxima de relación).

- Ajustar la estrategia comunicativa (foco en la cultura original o en la cultura término) al encargo de traducción (máxima de calidad y de relación).

- Utilizar sólo marcadores con los que esté familiarizado el lector (máxima de relación).

- Mantener solo las distinciones que el lector pueda apreciar (máxima de cantidad).

- No mantener en el TM distinciones del TO que no tengan una función comunicativa (por ejemplo, dialecto) (máxima de cantidad).

- Utilizar el mínimo de marcadores que, junto con otras pistas de contextualización, permita identificar los rasgos situacionales y crear

\footnotetext{
${ }^{14}$ Mayoral Asensio, Roberto, La traducción de la variación lingüística, Monográficos de la revista Hermeneus, 1, Soria, Diputación Provincial de Soria, 1999, p. 178.
} 
el efecto deseado (salvo en aliteración deliberada) (máxima de cantidad).

- No introducir ambigüedad injustificada en la definición de los rasgos situacionales (máxima de calidad).

- Evitar la incoherencia (en el caso de los parámetros culturales, mezclando rasgos propios de ambas culturas) (máxima de modo).

- Mantener la coherencia en el tipo de marcadores utilizados para señalar un rasgo determinado y el conjunto de los rasgos de un texto (máxima de modo).

Mayoral también apunta la subjetividad inevitable en este proceso. Marco ${ }^{15}$ explica los tipos de soluciones que pueden darse a distintos problemas en cuanto a la traducción de las variantes dialectales en torno a diversos ejes:

a) Con marcas/sin marcas. La primera solución consistiría en intentar, de forma parcial o total, reproducir los rasgos dialectales del original. Esto se puede conseguir transgrediendo la norma lingüística de la LM en alguno de sus niveles (ortográfico, gramatical, léxico) o no. La segunda, se refiere a la traducción neutralizada de la variación dialectal o a la anulación de la misma. Se sustituiría por la variedad estándar de la LM.

b) Con transgresión/sin transgresión. La primera solución podría incluir técnicas como la elisión de vocales o consonantes, la transcripción como /u/ de todas las /o/ átonas, el uso de estructuras incorrectas o vocablos no aceptados por la lengua estándar, etc. En el segundo caso, se evitarían las soluciones que violen la norma lingüística y sí incluirían la informalidad para sustituir un determinado dialecto o el estilo oral.

c) Naturalidad/convencionalidad. En el primer caso, se optaría por elegir un dialecto particular para la traducción o, por el contrario, crear una configuración artificial de rasgos de diversa índole que no recuerde a ningún otro dialecto vigente en la cultura meta.

El autor insiste en que no hay recetas simples pero que a pesar de las pérdidas evidentes a las que el traductor se expone, una pérdida parcial será siempre preferible a una total.

Finalmente, Ramos ${ }^{16}$ analiza las técnicas de traducción del dialecto geográfico que aparece en la obra teatral Pygmalion de G. B. Shaw a las versiones portuguesas, tanto para ser publicadas en papel como para el

${ }^{15}$ MARco Borillo, Josep. El fil d'Ariadna. Anàlisi estilística i traducció literaria, Barcelona, Eumo, 2002, pp. 80-86.

${ }^{16}$ RAMOS PINTO, Sara. "How important is the way you say it?", Target, 21:2, 2009, 289-307. 
teatro y la televisión. Esta autora organiza las técnicas de traducción en torno a dos decisiones básicas: la de preservar la variedad lingüística o la de no hacerlo. Si se decide no hacerlo se puede optar por la normalization, o traducción a una variedad estándar en todo el texto, o por la dialectization, que supondrá traducir enteramente a una variedad no estándar. Si, por el contrario, la intención del traductor es la de preservar el dialecto, entonces tendrá que elegir entre:

1) conservar las coordenadas de espacio y tiempo en el TM utilizando elementos no estándar familiares para los lectores meta, lo que incluye: a) añadir coletillas que indiquen que el personaje habla en dialecto; b) reducir el dialecto a los tratamientos; c) subir el nivel de formalidad del discurso estándar; d) utilizar rasgos discursivos orales; e) utilizar rasgos (léxicos, morfosintácticos, gráficos o fonéticos) de otras variedades y f) utilizar rasgos (léxicos, morfosintácticos, gráficos o fonéticos) de una variedad específica, o;

2) conservar sólo las coordenadas de tiempo en el TM para lo que recurrirá al uso de elementos que no sean familiares para el lector, como a) importar directamente ciertos elementos léxicos del TO; b) introducir léxico del TO, pero adaptándolo a la ortografía de la LM y c) inventar un dialecto exclusivo para esa traducción;

3) no preservar las coordenadas de espacio y tiempo del TO. El traductor podrá recurrir al uso de rasgos (léxicos, morfosintácticos, gráficos o fonéticos) comunes en el lenguaje oral de la LM o subir el nivel de formalidad del discurso estándar;

4) no preservar las coordenadas de espacio del TO, pero sí las de tiempo, entonces se podrá hacer uso de rasgos (léxicos, morfosintácticos, gráficos o fonéticos) de diferentes variedades lingüísticas o de una específica.

De todas las técnicas que lista, la autora afirma que

probably because translators often have no specialized linguistic knowledge of their own language and work with stereotypical features easily recognized by the target context community, a combination of different strategies is often found in the same translated text.

Resumiendo, las técnicas de traducción propuestas por estos autores se pueden sintetizar en la traducción hacia un dialecto de la lengua meta, la traducción hacia la lengua estándar, la traducción a través de elementos coloquiales de diverso tipo y la creación de rasgos dialectales ad hoc. 


\section{FUNCIÓN DE LOS DIALECTOS EN LITERATURA}

El dialecto es un artificio más en el abanico de herramientas estilísticas que enriquecen la obra. El dialecto existe en la literatura, pero también existe la literatura en dialecto (Brook, 1978: 184). La diferencia entre ambas expresiones radica en la aparición limitada al habla de uno o más personajes, en el primer caso, o en el protagonismo del dialecto como el vehículo lingüístico por el que se cuenta la historia, en el segundo. En cualquiera de las dos situaciones, el dialecto hace su aparición en una novela cuando el autor decide hacer que su narración o diálogos se viertan según la variante de un área geográfica concreta, una clase social, una época determinada o una forma de hablar particular. No cabe duda, entonces, de que la inclusión de uno o más dialectos por parte del autor es algo intencionado.

Tan importante como el dialecto en la literatura es su finalidad. Mair ${ }^{17}$ indaga en las dos funciones principales que según él subyacen al dialecto en la literatura: de un lado, la función mimética, que hace que el dialecto refleje la realidad del hablar de una comunidad lingüística; aquí el dialecto es el espejo en el que se refleja una sociedad o la situación que vive la misma. El autor es un observador y los personajes son los actores que se expresan de una forma muy parecida a la del dialecto real, por lo que el objeto de éste se torna costumbrista, como la foto de una realidad en movimiento.

De otro lado, Mair propone la función simbólica, donde la variante dialectal le sirve al autor para expresar su manera de ver la vida de forma crítica. El dialecto tiene poder caracterizador, o dicho de otro modo, la forma de hablar de un personaje simboliza lo que es. Por lo tanto, la variación dialectal puede ser una herramienta para establecer oposiciones entre los personajes. Esto le permite al autor ironizar, trasmitir su rechazo o ridiculizar sobre un tipo de persona, situación o sociedad. El dialecto tiene aquí una función distanciadora y estigmatizadora. El efecto en el lector es muchas veces el humor que provocan algunos personajes caricaturescos que se manifiestan de forma distinta al resto y que con su forma de hablar proyectan una ideología determinada: la de la incultura, las clases bajas o los esnobs, entre otros. El rechazo, la condescendencia o la antipatía son de igual manera reacciones buscadas en ciertas formas de hablar, con lo cual el dialecto no sólo ofrece una muestra de una forma de expresarse, sino que dibuja un modo de sentir que pretende mover sentimientos en los lectores.

\footnotetext{
17 MAIR, Christian, "A methodological framework for research on the use of nonstandard Language in fiction", en Arbeiten aus Anglistik und Amerikanistik, vol. 17, 1, 1992, pp. 103-123.
} 
La función mimética puede utilizarse con visos de otorgar la máxima verosimilitud a la historia que se cuenta, pero también para identificarse con una comunidad de hablantes. En ocasiones, el dialecto de la novela es el propio del autor, el cual, a través de él, pretende dejar patente su pertenencia a una comunidad lingüística, su solidaridad y su reivindicación de la valía de ésta por medio de uno o más personajes. Se dan casos en los que esta intención se plasma construyendo novelas enteramente escritas en dialecto, donde la función política puede primar sobre la literaria. Así, el creador se acerca a sus personajes y crea un vínculo entre la invención y su propia persona.

En la función simbólica, sin embargo, el personaje o los personajes se manifiestan en una lengua diferente a la del narrador, lo que hace que autor y lectores contemplen a los personajes desde fuera, como espectadores de una representación. Pero las intenciones del autor son variadas y esto no siempre es así, ya que los personajes que hablan en dialecto pueden encarnar valores defendidos por el autor y entonces son los personajes malvados, fríos o convencionales los que se expresan a través de la lengua estándar. Existen ejemplos célebres del dialecto en literatura como el habla del guardabosques del norte de Inglaterra de Lady Chatterley's Lover o el esclavo Jim en The Adventures of Huckleberry Finn. Estos casos vienen a constatar, además, que la mezcla de las dos funciones está presente en los dialectos recreados, pudiéndose dar una en mayor medida que la otra, pero conteniendo en la mayoría de ocasiones rasgos de las dos.

Lo que es más relevante para el presente trabajo es que, al dotar a un personaje de un habla dialectal, el autor lo está destacando, le está dando importancia. El autor pretende, por un lado, que esa forma de hablar sea inteligible, por lo que estos dialectos son siempre una recreación más o menos fidedigna de la realidad; por otro, siempre existe una voluntad estilística en su creación, por lo que el escritor puede seleccionar rasgos correspondientes a un dialecto real o inventar uno para su obra. Descubrir la función del dialecto, cuando el fin es la traducción, es un paso fundamental en el análisis de la obra y probablemente la clave para determinar técnicas de traducción adecuadas.

\section{LA TRADUCCIÓN LITERARIA EN EL SIGLO XX}

Si bien no es objeto de este artículo profundizar en un recorrido histórico sobre los acontecimientos por los que pasó la traducción literaria en España hasta la actualidad, un breve repaso de su historia en el siglo XX servirá para contextualizar la mayor parte de las traducciones que aquí se analizan. 
Es importante destacar el papel de Francia en la historia de la traducción española, pues desde el siglo XVII, la lengua francesa empieza a adquirir protagonismo. Por una parte, se implanta un predominio de la literatura francesa como proveedora de obras originales, y, por otra, el francés se convierte en idioma intermediario entre los textos que vienen del inglés, del alemán o del neerlandés. Como apunta Pym ${ }^{18}$, esta preferencia continuará durante dos siglos más, en los que las obras francesas también serán las más traducidas y ese país dictará modas literarias y conductas traductoras. Sin embargo, según el mismo autor, a principios del siglo XX los autores alemanes e ingleses "also entered Castilian after their acceptance in France, although the translations were increasingly from the original languages". En 1910, la Biblioteca Nueva acoge las traducciones de numerosos autores europeos, eso sí, la mayor parte franceses.

Bouza $^{19}$ defiende la abundante publicación de obras inglesas en castellano y viceversa. Según él, en el siglo xix ya existía una actividad editorial llena de vitalidad que suponía un vehículo para el intercambio cultural entre España y el Reino Unido. De las primeras décadas del siglo $\mathrm{XX}$, Pym destaca a dos personajes significativos por lo que se puede extraer de sus acciones en torno a la traducción: Luís Astrana Marín, quien traduce las obras completas de Shakespeare en 1929, y José Ortega y Gasset, el que escribe Miseria y esplendor de la traducción (1937), famoso ensayo formulado durante la Guerra Civil española.

La dictadura franquista posterior impuso una censura sobre la traducción y sobre los traductores, ya que muchos de ellos eran profesores, periodistas 0 intelectuales exiliados a Europa o América. Ortega ${ }^{20}$ explica así la situación literaria en España después de la Guerra Civil:

La falta de creación literaria en lengua española durante los primeros años de la dictadura franquista, debida a la muerte, encarcelamiento y exilio de un gran número de escritores españoles, supuso un aumento considerable de las traducciones. La falta de libre expresión obligó a muchos hombres y mujeres de letras a abandonar las carreras que desarrollaban antes de la Guerra Civil y a dedicarse al oficio de la traducción. Asimismo, los editores reclutaban traductores, profesionales o no, para suplir la carencia de autores de expresión española.

\footnotetext{
${ }^{18}$ PYM, Anthony. "Spanish tradition", en M. Baker (ed.) Routledge Encyclopedia of Translation Studies. London: Routledge, 1998, 552-563.

${ }_{19}$ BouzA, Fernando, Anglo-Hispana: Five Centuries of Authors, Publishers and Readers Between Spain and the United Kingdom. Madrid: Ministerio de Cultura, Secretaría General Técnica, Subdirección General de Publicaciones, Información y Documentación, 2007, p. 78

20 OrTEGA SÁEZ, Marta, "Juan G[onzález-Blanco]. de Luaces: el traductor desconocido de la posguerra española”, en Arbor. Ciencia, pensamiento y cultura, vol. 185, 740, 2009, pp. 1339-1352.
} 
La censura también actuó implacablemente sobre la diversidad lingüística de las provincias españolas, pues sólo era posible traducir hacia el castellano y bajo los términos y condiciones que el régimen imponía. También por razones políticas, Francia pierde importancia como lengua de la que se traducía a favor de Alemania. Sin embargo, Vega ${ }^{21}$ afirma que "el grueso de la traductografía lo constituían los autores de la anglofonía", tendencia ésta última que se mantiene hasta nuestros días. De hecho, en el primer tercio del siglo XX, según documenta Bouza, ya existe una vitalidad editorial en la que algunas editoriales como La España Moderna de José Lázaro Galdiano se embarcan en una política ambiciosa de traducciones del inglés. Pero si las traducciones del francés van cediendo al protagonismo creciente de las del inglés, Pym argumenta que conforme avanzan los años 60 , "el denominado espíritu sesentayochista también penetró en nuestro país con relativa presteza gracias a la frenética actividad traductora y editorial que hacia esos años se desata en España".

La llegada de la democracia en 1975 supone una dosis de libertad en lo que se refiere a traducción, puesto que muchas obras antes prohibidas se tradujeron y las lenguas peninsulares diferentes al castellano empezaron a resurgir. La traducción se vuelve a alentar desde todos los frentes y hacia las lenguas españolas. Pym rescata un dato sobre la traducción en España en 1992: en este año, la literatura traducida supone un $26 \%$ del total de obras publicadas, un porcentaje algo superior a la media internacional.

\section{CUMBRES BORRASCOSAS}

Este estudio continúa con el acercamiento a las técnicas de traducción del dialecto de la novela Wuthering Heights (Emily Brontë, 1847) al castellano en cinco momentos diferentes del siglo XX y XXI. Un paso previo al análisis de algunos fragmentos traducidos al castellano será el estudio de la obra original, no incluido en este trabajo por razones de espacio. Sí es importante mencionar la naturaleza del personaje a través del cual se hace patente el dialecto en la novela: Joseph. Se trata del criado de la casa de la familia Earnshaw, una persona de clase baja, sin educación y profundamente religiosa. Joseph es temido y odiado a partes iguales, pues sus palabras son siempre despreciativas y malintencionadas. El viejo criado representa a la sociedad conservadora de los pueblos rurales ingleses de esa época y, a pesar de ser un personaje secundario, se encuentra presente en todas las encrucijadas que acontecen al resto de personajes.

\footnotetext{
${ }^{21}$ VEGA, Miguel Ángel. "De la guerra civil al pasado inmediato", en Francisco Lafarga y Luis Pegenaute (eds.) Historia de la traducción en España, Salamanca, Ambos Mundos, 2004, p. 544.
} 
Una obra de la categoría de Cumbres borrascosas ha pasado, lógicamente, por las manos de muchos traductores y editores que la han querido acercar al público español. La primera traducción al castellano de la novela se realizó en 1921 gracias a Cipriano Montolín. A partir de ésta, los nombres que se vuelven a hacer cargo de la conocida obra son muchos. Las editoriales también son varias y la mayoría relanza la obra en varias ocasiones. Las últimas versiones de Cumbres borrascosas al castellano son las publicadas en 2010 (una a cargo de RBA, colección Grandes escritoras, que aprovecha la traducción de Carmen Martín Gaite con prólogo de la escritora Carmen Posadas y otra editada por Aldevara con traducción de Juan González Blanco) y en 2011 (una reedición de Alianza Editorial que retoma la traducción de Rosa Castillo). El presente análisis gira en torno a cinco de las traducciones de la obra de Brontë al castellano realizadas en diferentes años: 1942, 1970, 1984, 1989 y 2007, correspondientes a los traductores Juan G. de Luaces, E. Reguera, Carmen Martín Gaite, Rosa Castillo y Cristina Sánchez-Andrade.

La primera de ellas es obra del traductor Juan González-Blanco de Luaces, según Ortega ${ }^{22}$ (2009: 1341) "el traductor más prolífico de la posguerra española". Tradujo obras del portugués, del inglés y del francés y se encargó de autores como Dickens, Charlotte y Emily Brontë, Dostoievsky, Kipling o Swift. La editorial que presenta la obra, la catalana Destino se inició en la década de los 40 del siglo xx. La edición que se estudia aquí de 1973 (séptima edición a raíz de la primera, publicada en 1942) se inserta dentro de la colección Áncora y Delfín, número 2, y cuenta con un prólogo anónimo en el que se ofrecen datos sobre la autora, la novela y la gestación de la misma. Además de esto, no existe ningún rastro o visibilidad del traductor más allá del propio texto en castellano y de la traducción de los nombres propios de los personajes siempre que le ha sido posible.

La segunda traducción objeto de estudio la publica la catalana Ediciones Rodegar en 1970 y vuelve a hacerlo tres años más tarde. E. Reguera, el traductor, traslada al castellano otro trabajo de las hermanas Brontë en el mismo año: Jane Eyre. Los datos encontrados sobre éste se limitan a su escasa bibliografía referenciada. A través de él, sin embargo, se tiene la oportunidad de conocer la postura traductora acerca de la prosa victoriana de Brontë en los años 70 del siglo XX. Esta traducción se presenta en una edición donde no se incluyen notas ni prólogo.

El siguiente texto de estudio será el de Carmen Martín Gaite, la traductora más conocida de Cumbres borrascosas y de la que más veces se ha tomado su texto para reeditarlo. Se cuenta con la edición de Debolsillo

${ }^{22}$ Op. cit. 
de 2005, en la cual no se incluye prólogo, aunque sí lo había en 1984, en la primera edición que publicó su versión y en una edición de 1987. Además de la novela, el género en el que Martín Gaite fue más prolífica, y el ensayo, cultivó la crítica literaria y la traducción. Entre sus autores traducidos se encuentran Emily Brontë, Charlotte Brontë, Gustave Flauvert, Rainer Maria Rilke o George McDonald.

El siguiente texto en este cotejo será el de Rosa Castillo, para el que se cuenta con un ejemplar de Alianza Editorial de 2005. Esta traductora (1911-2003) vierte el texto de Brontë para Cátedra en 1989 y un año después traduce los poemas de la autora inglesa para la editorial Torremozas. Por último, Cristina Sánchez-Andrade es una escritora gallega cuya traducción de Cumbres borrascosas se edita en 2007 y 2010 gracias a la editorial Siruela.

Para terminar con esta breve introducción a los traductores, cabe decir que ni Reguera ni Martín Gaite (al menos en las ediciones que se utilizan) introducen notas al pie en la novela; sí lo hace Castillo para aclarar asuntos culturales y citas bíblicas, de la misma forma que SánchezAndrade. Ésta última incluye una nota introductoria donde defiende su forma de traducir. Además, esta edición incluye un prólogo de Alejandro Gándara.

Los cinco fragmentos de las traducciones se muestran siguiendo su orden de antigüedad para facilitar la comparación cronológica de los textos: 1.a, G. de Luaces (1942); 1.b, Reguera (1970); 1.c, Martín Gaite (1984); 1.d, Castillo (1989), y 1.e, Sánchez-Andrade (2007). A continuación se ilustra qué modelo de lengua en castellano han elegido los traductores para con el dialecto Northern del norte de Inglaterra (zona de West-Riding) que se expresa en los diálogos de Joseph:

1) 'Yon lad gets war und war!' observed he on re-entering. 'He's left th' gate at $t$ ' full swing, and Miss's pony has trodden dahn two rigs o' corn, and plottered through, raight o'er into $t$ ' meadow! Hahsomdiver, $t$ ' maister 'ull play t' devil to-morn, and he'll do weel. He's patience itsseln wi' sich careless, offald craters - patience itsseln he is! Bud he'll not be soa allus - yah's see, all on ye! Yah mun'n't drive him out of his heead for nowt!'

(...)

'I sud more likker look for th' horse,' he replied. 'It 'ud be to more sense. Bud I can look for norther horse nur man of a neeght loike this as black as t' chimbley! und Heathcliff's noan t' chap to coom at MY whistle - happen he'll be less hard o' hearing wi' YE!' (Brontë, 2003 $\left.[1847]^{23}\right)$

${ }^{23}$ BRONTË, Emily. Wuthering Heights: the 1847 text, backgrounds and contexts criticism, R. J. Dunn (ed.), 2003, New York, Norton, 1847, p. 94. 
1.a) — ¡Cuánta guerra da ese muchacho! Ha dejado abierta la verja, y la jaca de la señorita se ha escapado a la pradera, después de estropear dos haces de grano. Ya le castigará el amo mañana por esos juegos endemoniados, y hará bien. Demasiada paciencia tiene al tolerar tantos descuidos. Pero no sucederá siempre igual. Todos lo hemos de ver. ¡Está haciendo todo lo posible para sacar al amo de sus casillas!

(...)

-Con más gusto hubiera buscado al caballo, y hubiera sido más razonable - respondió él-. Pero no puedo encontrar ni a uno ni a otro en una noche tan negra como la de hoy. $Y$ si silbo para llamarle, bien cierto es que no vendrá. Puede que no tuviera el oído tan duro si le silbara usted (Brontë, $1942^{24}$ ).

1.b) -Ese muchacho va de mal en peor - dijo al entrar- . Ha dejado abierta la verja y la jaca de la señorita se ha escapado, después de estropear dos haces de grano. El dueño mañana se pondrá furioso y con razón... Tiene demasiada paciencia..., pero me parece que ya se le está acabando... El chico hace todo lo que puede por sacarle de sus casillas...

(...)

- Más hubiera valido buscar el caballo... Pero ni caballo ni hombre pueden ser hallados en una noche tan lóbrega como ésta (Brontë, $\left.1970^{25}\right)$.

1.c) -Este chico no sirve más que para dar guerra y más guerra entró diciendo-. Ha dejado abierta la verja de par en par y la jaca de la señorita ha escapado a campo traviesa pisoteando el trigal y se ha ido derecha al prado. Seguro que mañana el amo le va a armar una de todos los demonios, y hará más que bien. Demasiada paciencia tiene con semejante manazas inútil, ¡demasiada! Pero no le va a durar siempre, y si no, al tiempo. ¡Ahora no hace falta mucho para que estalle!

(...)

- Más sensato hubiera sido buscar el caballo, y yo lo habría preferido - contestó-. Pero quién busca a caballo ni hombre alguno en una noche como ésta, negra como boca de lobo. Y de Heatcliff no se puede esperar que atienda a un silbido mío. Seguro que para el de usted sería menos duro de oído (Brontë, 2005 [1984] ${ }^{26}$ ).

\footnotetext{
${ }^{24}$ BRonTË, Emily. Cumbres borrascosas (trad. de Juan G. de Luaces). Barcelona: Destino, 1942 , p. 90

${ }^{25}$ BRONTË, Emily. Cumbres borrascosas (trad. de E. Reguera). Barcelona: Rodegar, 1970, p. 50.

${ }^{26}$ BRONTË, Emily. Cumbres borrascosas (trad. de Carmen Martín Gaite). Barcelona: Debolsillo, 2005 [1984], pp. 130-131.
} 
1.d) -Este chico va de mal en peor —observó al volver-. Ha dejado la verja abierta de par en par, y la jaca de la señorita ha pisoteado dos hileras de grano, y se ha ido derecha al prado. De todas maneras, el amo se pondrá como un diablo mañana, y le dará su merecido. Él tiene paciencia con estas criaturas descuidadas e inútiles, es la misma paciencia, pero esto no puede durar, ya lo verá usted, y todos. ¡No le sacaréis de quicio en vano!

(...)

- Hubiera sido mejor que hubiera buscado al caballo, hubiera sido más sensato, pero no puedo buscar ni al caballo, ni al hombre en una noche como ésta, más negra que una chimenea. $Y$ Heathcliff no es mozo que acuda a mi silbato, acaso sería menos duro de oído con usted (Brontë, $2005[1989]^{27}$ ).

1.e) Ese chico va de mal en peor -observó al volver a entrar- $\mathrm{Ha}$ dejado abierta la verja de par en par y la jaca de la señorita ha pisoteado el trigal y se ha escapado al prado. Seguro que mañana el amo le arma una buena, y se la tiene merecida. Demasiada paciencia está teniendo con semejantes criaturas descuidadas e inútiles, ¡demasiada! Pero no le va a durar para siempre, y si no, espera a ver. No le sacaréis de quicio en vano.

(...)

- Más nos habría valido buscar al caballo —respondió-. Más sensato habría sido, pero no puedo buscar ni al caballo ni al hombre en una noche como ésta, negra como una chimenea. Y Heathcliff no es rapaz que acuda a mi silbido..., puede que sea menos duro de oído con usted (Brontë, 2007 28 ).

La intervención anterior del personaje de Joseph en lengua original combina algunas características de la lengua oral (contracciones, puntuación) que comparten protagonismo con los rasgos fonológicos más propios del dialecto Northern ("war", "dahn", "raight", "itsseln"). El primer fragmento traducido (1.a) muestra una lengua estándar en castellano. Pero, más allá de eso, el discurso de Joseph, si bien es coherente con la forma de ser del personaje, incurre en diversos errores de traducción que podrían deberse a la falta de comprensión del original. Por ejemplo, "Cuánta guerra da ese muchacho" en "Yon lad gets war und war!", "Ya le castigará el amo mañana por esos juegos endemoniados" en "t' maister 'ull play t' devil tomorn" o "Demasiada paciencia tiene al tolerar tantos descuidos" en "He's patience itsseln wi' sich careless, offald craters". Además, el énfasis que Brontë pone a través de las exclamaciones queda matizado por la eliminación de algunas de ellas.

\footnotetext{
${ }^{27}$ BRONTË, Emily. Cumbres borrascosas (trad. Rosa Castillo), Madrid: Alianza Editorial, 2005, pp. 111s.
}

${ }^{28}$ BRONTË, Emily. Cumbres borrascosas (trad. Cristina Sánchez-Andrade), Madrid: Siruela, 2007, p. 116. 
En la traducción de Reguera (1.b) contrastan el registro quizá elevado para el personaje originalmente de clase baja, que se manifiesta en estructuras como "pueden ser hallados" y "lóbrega", con la expresión más coloquial "hace todo lo que puede por sacarle de sus casillas". El uso de los puntos suspensivos podría interpretarse como sustituto de las exclamaciones del texto original. En la lectura en castellano, esta tipografía confiere a la intervención de Joseph un tono algo amenazante, lo que encaja muy bien con el personaje. Sin embargo, este recurso se utiliza en detrimento de texto que se elimina ("sich careless, offald craters", "und Heathcliff's noan t' chap to coom at MY whistle - happen he'll be less hard o' hearing wi' YE!"), y por tanto, merma la agresividad y desprecio con que Joseph se dirige a sus interlocutores.

Martín Gaite (1.c) expresa en su texto una tímida voluntad por adecuar el registro a la clase social del personaje con expresiones como "al tiempo" o "duro de oído". No obstante, este intento es leve y podría resultar imperceptible para el lector. La traducción de Castillo (1.d), por su parte, incurre en alguna incongruencia de sentido en "Él tiene paciencia con estas criaturas descuidadas e inútiles, es la misma paciencia, pero esto no puede durar..."; en el estilo, al dirigirse de usted a sus amos para tutearlos seguidamente; o en alguna cuestión ortotipográfica.

La última traducción (1.e), de Sánchez-Andrade, puede resultar menos expresiva que el original al omitir, excepto en una ocasión, los signos de exclamación. Es importante la consideración de este recurso que aparece en el original, ya que da muestra del permanente estado de exaltación de Joseph. La expresión "negra como una chimenea", que ya utilizó Castillo, es una traducción literal del inglés.

Estas matizaciones conducen a afirmar que, ante la aparición dialectal en el texto original en forma de variedad geo-social, los traductores utilizan la técnica de traducción de neutralización del dialecto en castellano. Las cinco versiones, al elegir la lengua estándar para Joseph, se alejan en alguna medida del tenor o nivel de formalidad que exhibe el habla del personaje y que le caracteriza.

La comparación de las traducciones entre sí conduce a señalar los trabajos de G. de Luaces, Reguera, Castillo y Sánchez-Andrade como algo más deficientes que el de Martín Gaite debido a los repetidos errores de traducción en el primer caso y a las omisiones en el segundo. Castillo ofrece una traducción que resulta algo literal ("más negra que una chimenea", "amable y sencillamente") y Sánchez-Andrade logra una traducción parecida a ésta última.

Es importante el hecho de que la postura que han adoptado los traductores sea la misma, al coincidir en no adoptar ninguna técnica que pueda restituir el dialecto como hubiera podido ser la creación de rasgos 
subestándar de diversa índole, no característicos de un dialecto concreto. Utilizando términos de Toury, esta inclinación por la aceptabilidad, frente a la adecuación, podría denotar una falta de atención hacia el fenómeno de la traducción de la variación lingüística a pesar del tiempo transcurrido y de los diferentes traductores que han tratado el texto. De hecho, la traducción más reciente de este pequeño corpus, la realizada por Sánchez-Andrade ${ }^{29}$ en 2007, aporta datos claros en su nota a la traducción que introduce su texto:

\begin{abstract}
En el caso de esta obra hay algo que es absolutamente imposible de reflejar: el acento de la zona de Yorkshire (zona al noroeste de Inglaterra de donde procedían las hermanas Brontë y que se aprecia muy bien en películas como Full Monty o Little Voice) del personaje del criado Joseph, cuyos matices (esa sorna taciturna y seca) no tienen equivalente en castellano e inevitablemente se pierden. Es como si alguien pretendiera encontrar un equivalente en otro idioma al acento andaluz, o al acento murciano.
\end{abstract}

La afirmación de la traductora es rotunda y no cabe duda de que la decisión sobre el dialecto en este caso es personal, aunque siempre influenciada indirectamente por lo que se suele hacer en estos casos en la literatura traducida española.

\title{
5. TIEMPOS DIFÍCILES
}

Aunque no siempre fue así, hoy en día Hard Times (1854) es considerada una novela innovadora dentro de la temática dickensiana e imprescindible por su carácter histórico-social y por la inclusión funcionalmente motivada del dialecto. Estas innovaciones incluyen "a medley of voices" ${ }^{\prime 30}$, o dicho de otra manera, diferentes hablas presentes en el relato, sobre las que destaca el acento del norte de Inglaterra (zona de Lancashire) de uno de los personajes.

Para el análisis siguiente se va a recurrir, de un lado, a dos de las versiones más actuales de la obra de Dickens para el público adulto, realizadas en 1995 y 2005, y de otro, a la traducción que durante décadas se ha utilizado en España de la novela, obra de Amando Lázaro Ros (1949), y a una de las primeras versiones, la de José Camino Nessi (1921). El texto de Lázaro Ros (dentro de Obras completas, $2^{\circ}$ tomo, Aguilar) es el que más veces se ha reeditado de entre las traducciones realizadas de Hard Times al español, por encima de las de José Camino Nessi, José Méndez Herrera o José Antonio Vidal Sales.

${ }^{29}$ Op. cit.

30 FOWLER, Roger, "Polyphony in Hard Times", en Ronald Carter y Paul Simpson (eds.) Language, Discourse and Literature: An Introductory Reader in Stylistics, London: Unwin Hyman, 1989, p. 79. 
Escritor, traductor y periodista vasco, Lázaro Ros tradujo a autores como Zola, Robert Louis Stevenson, Jane Austen o Kipling, entre otros, y aunque volvió a traducir algún otro trabajo de Dickens, tuvo mayor continuidad con la obra de autores como Arthur Conan Doyle. José Camino Nessi fue un escritor y poeta precoz nacido en Filipinas. En 1921 traduce Tiempos difíciles, obra que se publica en la editorial madrileña Saturnino Calleja. Víctor Pozanco tradujo esta obra en 1995, cuando la publica la editorial Ronsel, único sello donde se halla disponible su traducción. Su relación con Dickens le lleva a contribuir con un prólogo en la traducción de Los perezosos, realizada por Jordi Gubern. Pozanco cuenta con una larga carrera en el mundo de la traducción, dedicándose a autores exitosos entre el gran público.

El versor del último texto objeto de comparación es Ángel Melendo, quien sí ha visto cómo su trabajo se reimprimía en diferentes ediciones (RBA, 2006, 2009 y 2010; Gredos, 2009). Es curioso que a pesar del buen funcionamiento de su traducción, no exista información sobre la trayectoria de este traductor aparte de su incursión en el mundo de Dickens. La editorial Gredos es un sello dentro del grupo RBA, por lo que la edición posterior de la traducción de Melendo por RBA da muestras de la rentabilidad que se pudo obtener de ella. La que se estudia aquí es la publicada por Gredos en 2005 dentro de la colección Biblioteca Universal y cuenta con una introducción de Dámaso López García. La versión también incluye numerosas notas al pie a cargo del traductor con las aclaraciones pertinentes a elementos culturales, referencias históricas y sobre la obra del autor. En la traducción de que se dispone de Lázaro Ros, corregida por Galván, las notas (también de este último) son abundantes. En cambio, Pozanco no incluye ninguna.

La historia de Hard Times se inscribe dentro de ese tipo de obras que en un primer momento y durante una época no reciben la atención que merecen. Es destacable el hecho de que no se conozcan los nombres de los dos primeros traductores de Tiempos difíciles $(1863,1876)$. Otro dato que llama la atención es la diversidad de títulos bajo los que se ha editado la obra tanto en España como en Sudamérica. Estos dos factores dan a entender que la novela, si bien traducida en numerosas ocasiones por ser obra de Dickens, ha sufrido también la inconsistencia a lo largo de su historia.

Uno de los dialectos que presenta Hard Times es el de Stephen, personaje procedente del norte de Inglaterra y operario de la fábrica del señor Bounderby. Stephen representa la honestidad, los principios y la mansedumbre. A continuación se cotejan cuatro fragmentos correspondientes a las traducciones de Camino Nessi, 1921 (1.a); Lázaro 
(1949), corregida por Galván, edición de 2009 (1.b); Pozanco, 1995 (1.c) y Melendo, 2005 (1.d):

1) Sir, I were never good at showin o't, though I ha had'n my share in feeling o't. 'Deed we are in a muddle, sir. Look round town - so rich as 'tis - and see the numbers o' people as has been broughten into bein heer, fur to weave, an to card, an to piece out a livin', aw the same one way, somehows, twixt their cradles and their graves. Look how we live, ans wheer we live, an in what numbers, an by what chances, and wi' what sameness; and look how the mills is awlus a going, and how they never works us no nigher to ony dis'ant object - ceptin awlus, Death. Look how you considers of us, and writes of us, and talks of us, and goes up wi' yor deputations to Secretaries o' State 'bout us, and how yo are awlus right, and how we are awlus wrong, and never had'n no reason in us sin ever we were born. Look how this ha growen an' growen, sir, bigger an' bigger, broader an' broader, harder an' harder, fro year to year, fro generation unto generation. Who can look on 't, sir, and fairly tell a man 'tis not a muddle?' (Dickens, $2001[1854]^{31}$ ).

1.a) - Señor, yo no me he dado nunca maña para las explicaciones, a pesar de la parte que me toca. Claro es que estamos en un atolladero. Vea usted la ciudad, y lo rica que es, y vea usted la gente que ha venido aquí para tejer, para cargar, para trabajar en la tarea sin ninguna comodidad, y siempre lo mismo, desde la cuna hasta el sepulcro. Vea cómo vivimos, y dónde vivimos, y en qué forma y siempre igual; observe cómo las fábricas están marchando siempre, sin hacer que avancemos ni un solo paso, como no sea hacia la muerte. Vea usted cómo nos considera; lo que escribe de nosotros, lo que habla de nosotros y cómo envía usted delegados a la Secretaría de Estado para decirle mal de nosotros, y cómo tiene usted siempre razón desde que nacimos. Vea, señor, cómo el mal va creciendo y creciendo, cada vez con más fuerza, cada vez más cruel, cada vez más difícil, año tras año y generación tras generación. ¿Quién puede verlo, señor, y decir con toda convicción que no es un atolladero? (Dickens, $1921^{32}$ ).

1.b) -Señor, aunque yo he tenido mi parte de sufrimientos, nunca tuve habilidad para exponerlos. Señor, vivimos metidos en un embrollo. Fijaos en nuestra ciudad..., con todo lo rica que es..., y ved la gran cantidad de personas que han tenido la idea de reunirse aquí para tejer, para cardar y para ganarse la vida, todos con el mismo oficio, de un modo u otro, desde que nacen hasta que los entierran.

${ }^{31}$ Dickens, Charles. Hard Times: An Authoritative Text, Contexts, Criticism, (edición de Fred Kaplan y Sylvère Monod). New York, W. W. Norton \& Company, 2001, pp. 172.

${ }^{32}$ DiCKENS, Charles. Tiempos difíciles (trad. de José Camino Nessi). Madrid: Editorial Saturnino Calleja, 1921, p. 182. 
Fijaos en cómo vivimos, en dónde vivimos, en qué apiñamiento y con qué uniformidad todos. Fijaos en cómo las fábricas funcionan siempre, sin que con ello nos acerquen más a ninguna meta determinada y distante..., como no sea la muerte. Fijaos en el concepto en que nos tenéis, en lo que escribís acerca de nosotros, en lo que decís de nosotros, en las comisiones que enviáis a los ministros con quejas de nosotros y en que siempre tenéis razón y jamás la tuvimos nosotros en todos los días de nuestra vida. Fijaos en cómo todas esas cosas han ido creciendo y creciendo, haciéndose más voluminosas, adquiriendo mayor amplitud, endureciéndose más y más, de año en año, de generación en generación. ¿Quién que se fije con atención en todo esto no dirá, si es sincero, que es un embrollo? (Dickens, 2009 $\left.[1949]^{33}\right)$.

1.c) -Nunca he sabido expresar muy bien estas cosas, pero tengo mi opinión, sir. Fíjese en la ciudad (tan rica como es) y vea a todos esos tejedores y cardadores, a quienes se trajo aquí para que se ganasen la vida, sin hacer otra cosa hasta la tumba. Fíjese en cómo vivimos, y dónde vivimos, y cuántos, con qué perspectivas, tan idénticas para todos. $Y$ fíjese en lo bien que van las fábricas sin que nosotros notemos ninguna mejora, viviendo hacinados hasta la muerte. Y fíjese en qué consideración nos tienen, lo que escriben de nosotros, lo que dicen, y lo que informan sobre nosotros en el Parlamento; que siempre tienen ustedes la razón y nosotros nunca, como si fuésemos incapaces de razonar. Y fíjese que esto va a peor, de mal en peor, que se hace más duro año tras año, de generación en generación. ¿Quién puede ver todo esto, sir, y no decir, en honor a la verdad, que es un desastre? (Dickens, $1995^{34}$ ).

1.d) - Señor, nunca supe expresarlo bien, aunque he tenido mi parte de sufrimiento. Estamos dentro de un cenagal, señor. Fíjese en la ciudad..., tan rica como es..., y vea el número de sus gentes, que han sido traídas aquí para tejer, para cardar y ganarse la vida, todas de la misma manera desde la cuna a la sepultura. Fíjese en cómo vivimos, y en dónde vivimos, y en el número que somos, y cuál es nuestra suerte, y cuán semejante es; y mire cómo las fábricas están siempre en marcha, y cómo nunca nos acercan a ningún otro sitio distante que no sea el de la muerte. Fíjese en cómo nos consideran, y escriben de nosotros, y hablan de nosotros, y van con sus delegaciones a los ministros de estado para tratar de nosotros, y cómo ustedes siempre están en lo cierto, y nosotros siempre estamos equivocados, y nunca nos ha asistido razón alguna desde que nacimos. Y fíjese cómo esto ha ido creciendo y creciendo, señor, haciéndose más y más grande,

\footnotetext{
${ }^{33}$ DiCKENS, Charles. Tiempos difíciles. Para estos tiempos (ed. de Fernando Galván y trad. de Amando Lázaro Ros). Madrid: Cátedra, 2009, pp. 267-268.

${ }^{34}$ DickENS, Charles. Tiempos difíciles (trad. de Víctor Pozanco). Barcelona, Ronsel, 1995, pp. 229s.
} 
más y más extenso, más y más duro de año en año, de generación en generación. ¿Quién que se fije, señor, no ha de decir sinceramente que es un cenagal? (Dickens, $2005^{35}$ ).

En los textos anteriores, Stephen está defendiendo su opinión sobre la situación de los trabajadores delante del amo de la fábrica, el cual le ha preguntado por el nuevo estado de desazón que se está dando entre los mismos. Los marcadores que aparecen en el texto para recrear el acento norteño de Stephen se basan en las incorrecciones gramaticales (propias de un personaje sin cultura) como en "you considers of us", el eye-dialect ("o"', "livin'”), los rasgos más propios de la lengua de la zona de Lancashire (modificación de los participios pasados como "growen" o pronunciación diferente en "awlus") y la frase idiolectal "we are in a muddle".

La traducción de Camino Nessi (1.a) no refleja la particular habla del obrero en lengua inglesa. La versión castellana muestra una lengua estándar, algo que hace que Stephen adquiera un registro más culto del que le corresponde. Quizá puede destacarse algún elemento léxico, como "maña", que contribuye, aunque muy sutilmente, a un hablar menos formal para este personaje. Stephen se dirige al dueño de la fábrica de usted, algo que no se hace, por ejemplo, en la traducción de Lázaro y que resulta importante para crear el nivel de formalidad adecuado de un obrero que se dirige a su jefe. La frase idiolectal propia de Stephen se traduce como "atolladero" y se mantiene esta traducción en las dos ocasiones que aparece.

El comentario sobre un nivel de formalidad mayor se ve reflejado e incrementado al hablar de la traducción de Lázaro (1.b). El tono con el que habla Stephen es bastante más culto que en el texto original (in what numbers" por "con qué apiñamiento", "haciéndose más voluminosas" o "bigger an' bigger, broader an' broader" por "adquiriendo mayor amplitud"). Este registro no sólo se utiliza en esta escena en la que Stephen se dirige a sus superiores, sino cuando habla con personas de su misma clase como su mujer. La estructura del TO en cuanto a orden, extensión y tipografía se mantiene en esta traducción.

En la aportación de Pozanco (1.c), sin embargo, se mantiene en castellano el tratamiento de sir, con el que Stephen se dirige en la versión original al amo de la fábrica. En esta traducción también la criada del amo, la señora Sparsit, se dirige a él de esta manera. Sir es un término de cortesía inglés que debería haberse traducido como señor, puesto que la palabra en inglés sólo cabría conservarse si el sentido aludiera a la posesión de un título nobiliario. Además, el tratamiento delante de los

${ }^{35}$ DıCKENS, Charles. Tiempos difíciles (trad. de Ángel Melendo Gracia). Madrid: Gredos, 2005, p. 225. 
nombres que reciben los personajes en esta traducción se deja también en inglés (Mrs. Sparsit, Mr. Bounderby, etcétera). A falta de un prólogo, introducción o notas al respecto, se desconoce el motivo para conservar estos términos. Por otro lado, el tono que utiliza el traductor para el discurso de Stephen delante del señor Bounderby está más acorde con la naturaleza del obrero, un personaje humilde y casi analfabeto, lo que se evidencia en frases como "...esto va a peor, de mal en peor". La estructura oracional y la puntuación se alteran levemente para acomodarse al tono pretendido en castellano. El traductor realiza cambios cuando traduce "sin ever we were born" por "como si fuésemos incapaces de razonar", que no alteran el sentido del mensaje de Stephen. La frase recurrente de Stephen "tis a muddle; we are in a muddle" se omite en primer lugar y en segundo se traduce por "desastre". Precisamente la reiteración de esta expresión la convierte en un elemento idiolectal representativo de la situación del personaje.

Esta misma frase es tomada por Melendo (1.d) como "estamos dentro de un cenagal" y mantenida en las dos ocasiones en las que hace aparición. En cuanto al registro, esta traducción estaría entre las dos anteriores porque, sin dejar de mostrar un discurso formal, no llega a serlo tanto como en la traducción de Lázaro, donde se encuentran expresiones que pueden resultar poco creíbles en Stephen.

De la revisión de las cuatro muestras de traducción, es evidente que 1.a, 1.b y 1.d se ciñen más al TO que 1.c, donde el traductor indaga de otra forma en el discurso para amoldarlo a la idiosincrasia de Stephen. No obstante, las cuatro traducciones obvian la variación dialectal social y geográfica del personaje y le hacen comunicarse con un lenguaje estándar sólo alterado por su frase con carácter idiolectal y por el sir utilizado como vocativo (en 1.c), como únicas ayudas lingüísticas que le diferencian de los personajes de clase alta. Aunque Galván ${ }^{36}$ argumentaba que la variación lingüística que introduce Dickens en el personaje de Stephen es más bien una suerte de lengua coloquial, ninguno de los traductores ha tomado esta importante caracterización y ha aplicado una técnica no neutralizadora. Es obvia la mayor literalidad de las primeras versiones (Camino Nessi, 1921, y Lázaro, 1949). Esta literalidad se explica menos en la cuarta y más reciente versión (Melendo, 2005), parecida a la realizada por Lázaro, aunque el estilo de Melendo sea más pulido, intentando acercarse más al personaje. En la traducción de Pozanco el modelo de lengua es más llano, menos rimbombante y más cercano, lo que, como ya se ha dicho, encaja más con la forma en la que se expresaría Stephen.

${ }^{36}$ Op.cit. 
Stephen carece en nuestra lengua de ese lenguaje descriptor de su persona y clase, que, a su vez, representa un fresco de la clase obrera en una Inglaterra que comienza a industrializarse y donde se empiezan a ver las desigualdades que existen en el mundo de las grandes fábricas. Cabe pensar que tras muchas traducciones realizadas al castellano de esta obra los traductores hayan optado por no alterar el texto en castellano, llevados por el peso de lo que otros hicieron antes que ellos; sin embargo, la oportunidad de hacerlo sin duda hubiese añadido valor y riqueza a la traducción.

\section{CONCLUSIÓN}

Los dos ejemplos de obras con dialecto anteriores se han elegido por su representatividad, al tratarse de dos clásicos universales cuya antigüedad hace que hayan sido traducidos numerosas veces a lo largo del tiempo. Siendo el aspecto diacrónico central en el estudio, las obras clásicas son las más representativas en un trabajo de este tipo por la cantidad de versiones traducidas que aportan.

En esta escueta investigación, sólo dos de los traductores incluyen una introducción o nota a la traducción, donde explican las opciones elegidas para aspectos que consideran relevantes en la obra. Estas notas de descargo permiten a los traductores defenderse y justificar sus técnicas de traducción y el estilo elegido. Sin la ayuda de los testimonios de los traductores, en este análisis se ha intentado ilustrar cómo el paso del tiempo (y el consecuente avance de la Traductología) no ha influido positivamente, al menos en la traducción de dos novelas del siglo xix, inglesas y de fama mundial, en un cambio de actitud y de conducta hacia la problemática de la traducción de la variación dialectal por parte de traductores, editores y, en definitiva, del polisistema que los integra. La tendencia derivada de este estudio, en vista de los resultados analizados en las traducciones de 2005 y 2007 y en este tipo de novelas clásicas, se vuelca hacia la aceptabilidad, es decir, que predomina la voluntad de que el gran público siga accediendo fácilmente a la lectura de obras clásicas, sin elementos que puedan interrumpir una lectura cómoda.

El supuesto hecho de que los traductores anteriores no hayan sido conscientes del potencial que aporta la variación lingüística (o siéndolo, hayan decidido no actuar en beneficio de su restitución), conduce a aventurar las posibles causas de esta neutralización en tres. En primer lugar, que intencionadamente hayan optado por no reproducir el dialecto, a sabiendas de lo que representa. En segundo, que al tratarse de dos obras universales que cuentan con traducciones anteriores hayan sufrido la influencia de las versiones ya hechas y el temor a alterar lo que ya ha tenido aceptación de una determinada manera haya pesado sobre lo demás. 
Finalmente, que, más allá de eso, la tradición reinante en nuestro país hacia la neutralización del dialecto en las traducciones haya confluido con las dos posibles causas anteriores para que estos traductores hayan optado por la técnica de traducción de la neutralización.

La somera mirada dedicada más arriba a la historia de la traducción española podría dar pistas sobre las razones culturales, económicas, políticas, etc. que hacen que determinada técnica de traducción sea la más frecuente a la hora de traducir el dialecto al castellano durante décadas pasadas. A la luz de los hechos históricos acontecidos en España durante el siglo XX, no parece probable que estos hayan impulsado de alguna manera la traducción del dialecto original hacia la lengua estándar en las versiones estudiadas; sí parece más factible que las características del polisistema español como fuerza que dirige tradiciones y modas en literatura traducida arrastrara a editores y traductores hacia la neutralización a la lengua estándar de las diferentes voces en las obras.

El hallazgo sobre las normas de uso que se deriva de esta comparación es el de la inmovilidad de éstas y de la conducta traductora para con este problema de traducción a pesar del paso del tiempo. Un corpus amplio, donde se compararan las traducciones al castellano con originales ingleses de diferentes estadios históricos, sí podría dar una visión mucho más fiel sobre lo que aquí sólo se esboza. 